\title{
Urinary tract infections due to Amp C $\beta$-Lactamase producing gram negative bacteria
}

\author{
Dr Sangeetha $\mathbf{K}^{1}$, Dr Rudresh $\mathbf{S M}^{2}$, Dr Ravi GS ${ }^{3}$, Dr Raksha $\mathbf{Y}^{4}$
}

${ }^{1}$ Senior Resident, Department of Microbiology, ESIC Model Hospital, Asramam, Kollam - 691002, Kerala
${ }^{2}$ Associate Professor, ${ }^{3}$ Professor, ${ }^{4}$ Professor and Head, Department of Microbiology
ESIC MC \& PGIMSR, Rajajinagar, Bangalore-560010

DOI - 10.46319/RJMAHS.2019.v02i01.005

\section{Abstract}

Introduction: The exact occurrence of extended spectrum $\beta$-lactamase (ESBL) and AmpC $\beta$-lactamase production among uropathogenic bacteria is not known. Infections with drug resistant urinary pathogens have limited therapeutic options. Hence this study was conducted to know the occurrence of AmpC $\beta$-lactamase production among gram negative bacteria (GNB) causing urinary tract infection (UTI). Materials and Methods: The urine samples submitted to Department of Microbiology from 1st March 2016 to 30th April 2016 were studied. All the samples were plated on sheep blood agar and MacConkey's agar. The isolates were identified and Antibiotic susceptibility testing was done. AmpC $\beta$-lactamase and ESBL detection was done as described previously. The results were tabulated. Results: This study was conducted on 900 urine samples. Total 144 (16\%) GNB were isolated. E coli was the most common isolate followed by non-fermenting gram negative bacteria (NFGNB). AmpC $\beta$-lactamase production was seen in $75(52.1 \%)$ isolates and ESBL production was seen in 60 isolates $(41.7 \%)$. Total 27 (18.7\%) isolates showed multidrug resistant (MDR) phenotypes and 23/27 were AmpC $\beta$-lactamase producers. Conclusion: Routine detection of ESBL and AmpC $\beta$-lactamase is required to choose appropriate therapy. Judicious use of antibiotics should be done in clinical settings. Imipenem can be used as an empirical antibiotic for complicated urinary tract infection.

Keywords: AmpC $\beta$-lactamase, urinary tract infection, ESBL, multidrug resistance, E coli

\section{Introduction}

Urinary tract infection (UTI) is the commonest infectious disease at medical outpatient department. Infections by drug resistant urinary pathogens have very limited therapeutic options. ${ }^{[1]}$ The occurrence of AmpC $\beta$-lactamase among the urinary pathogens has limited therapeutic options and its production among urinary pathogens leads to the inoculum effect on the urinary mucosa further increasing the drug resistance. ${ }^{[2]}$

The AmpC $\beta$-lactamases belongs to class $C$ of Amblers classification. ${ }^{[1]}$ They have serene at their active site and are located in the bacterial periplasm. They are active on penicillins, cephalosporins, cephamycins and monobactams. They have very low activity against cefepime and carbapenems. Clavulanic acid inhibits class A enzymes but fails to act on AmpC $\beta$-lactamase. Sulbactam and tazobactam are also less effective against AmpC $\beta$-lactamase. ${ }^{[1]}$
Plasmid mediated AmpC $\beta$-lactamases were first detected in 1989 and are divided into various families like ACC, ACT, CMY, DHA, FOX and MOX based on amino acid sequences. ${ }^{[1-3]}$ These are constitutively produced except few which can be induced. Multiple resistance genes can be carried simultaneously with AmpC $\beta$-lactamase on the same plasmid. ${ }^{[1,4]}$ Strains carrying these genes were isolated from prolonged hospitalization, cancer patients, and rehabilitation centers. ${ }^{[5]}$ Risk factors for infection by such strains include admission to critical care unit, presence of indwelling catheter and prior antibiotic usage and treatment with $\beta$-lactamase inhibitor combinations. ${ }^{[4,5]}$

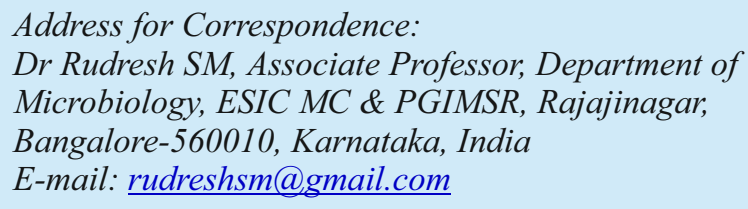


Hence the study was undertaken to know the occurrence of AmpC $\beta$-lactamase production among the GNB isolated from UTI and to know their sensitivity pattern.

\section{Materials and Methods:}

This is a prospective study carried out at ESIC MC \& PGIMSR, Bangalore, a tertiary care teaching hospital from $1^{\text {st }}$ March 2016 to $30^{\text {th }}$ April 2016. 144 GNBs isolated from 900 urine samples were identified to species level by Standard Laboratory techniques. ${ }^{[6]}$

Antibiotic sensitivity test was done according to CLSI 2015 guidelines. Briefly, a 0.5 McFarland suspension of the isolate was lawned on Mueller-Hinton agar (MHA) plates. The antibiotic discs like nitrofurantoin $(300 \mu \mathrm{g})$, ciprofloxacin $(5 \mu \mathrm{g})$, ceftazidime $(30 \mu \mathrm{g})$, cefotaxime $(30 \mu \mathrm{g})$, cefoperazone $(75 \mu \mathrm{g})$, piperacillin $(100 \mu \mathrm{g})$, piperacillin-tazobactam $(100 / 10 \mu \mathrm{g})$, and cefoperazone sulbactam $(75 / 10 \mu \mathrm{g})$ were placed and incubated overnight at $37^{\circ} \mathrm{C}$. The inhibition zone was interpreted according to CLSI guidelines. ${ }^{[7]}$

ESBL detection: Ceftazidime (CAZ: $30 \mu \mathrm{g})$ and Ceftazidime-clavulanic acid (CAZ/CAC; 30/10 $\mu \mathrm{g}$ ) discs were placed on lawn culture of the test organism. An increase in the zone of inhibition of CAZ/CAC by $\geq 5 \mathrm{~mm}$ was taken as ESBL production. ${ }^{[7]}$
AmpC $\beta$-lactamase detection: It was done according to Black et al. Briefly, E coli ATCC 25922 was lawn cultured on MHA plate along with a $30 \mu \mathrm{g}$ cefoxitin disc at the centre. A loop full colony of test isolate was inoculated to sterile filter paper strips impregnated with Tris-EDTA and was placed adjacent to cefoxin disc. After 18 hours of incubation flattening or indentation of the zone of inhibition was interpreted as AmpC $\beta$ lactamase positive. ${ }^{[8]}$

\section{Statistical analysis:}

Simple frequencies were tabulated and statistical significance was determined by Chi square test. A p value of less than 0.05 was considered statistically significant.

\section{Results:}

144 GNB were isolated from 900 urine samples. The antibiotic susceptibility pattern of the isolates is shown in Table-1. The organisms resistance to ciprofloxacin (71.5\%) followed by cefotaxime (70.8\%) and higher sensitivity was noted for imipenem $(3.5 \%)$ and amikacin (9\%). Among the organisms E.coli had higher antibiotic resistance compared to other Enterobacteriaceae and NFGNB.

Table -1 : Percentage distribution of antibiotic resistance among the isolates

\begin{tabular}{lcccccccccc}
\hline \multirow{1}{*}{ Organism } & CIP & CTX & NX & AMC & COT & NIT & PIT & GEN & AK & IPM \\
& $\%$ & $\%$ & $\%$ & $\%$ & $\%$ & $\%$ & $\%$ & $\%$ & $\%$ & $\%$ \\
\hline E. coli & 84.6 & 82.7 & 82.7 & 73.1 & 66.3 & 27.9 & 43.3 & 28.8 & 5.8 & 3.8 \\
Other Enterobacteriaceae & 37.1 & 40.0 & 31.4 & 45.7 & 60.0 & 62.9 & 22.9 & 17.1 & 17.1 & 2.9 \\
NFGNB & 40 & 40 & 40 & 40 & 60 & 100 & 20 & 40 & 20 & 0 \\
Total & 71.5 & 70.8 & 68.8 & 65.3 & 64.6 & 38.9 & 37.5 & 26.4 & 9.0 & 3.5 \\
\hline
\end{tabular}

Table - 2: Organism wise distribution of AmpC $\beta$-lactamases

\begin{tabular}{lcccccc}
\hline \multicolumn{1}{c}{ Organism } & \multicolumn{7}{c}{ AmpC } \\
& Positive & $\%$ & Negative & $\%$ & Total & $\%$ \\
\hline E. coli & 55 & 52.9 & 49 & 47.1 & 104 & 100 \\
Klebsiella spp & 7 & 58.3 & 5 & 41.7 & 12 & 100 \\
Citrobacter spp & 5 & 50.0 & 5 & 50.0 & 10 & 100 \\
Proteus spp & 2 & 25.0 & 6 & 75.0 & 8 & 100 \\
Enterobacter spp & 2 & 40.0 & 3 & 60.0 & 5 & 100 \\
NFGNB & 3 & 75.0 & 1 & 25.0 & 4 & 100 \\
Morganella morganii & 1 & 100.0 & 0 & 0.0 & 1 & 100 \\
Total & 75 & 52.1 & 69 & 47.9 & 144 & 100 \\
\hline
\end{tabular}


Table 2 shows the organism wise distribution of AmpC $\beta$-lactamases. The AmpC $\beta$-lactamase production was more commonly seen among E.coli $(\mathrm{n}=55 ; 73.3 \%)$ followed by Klebsiella spp and Citrobacter spp. The AmpC $\beta$-lactamase production was more common $52.1 \%$ followed by ESBL (41.7\%) and inducible AmpC $\beta$-lactamase (iAmpC; 8.3\%) (Table-3). Twenty four

Table - 3: Occurence of AmpC $\beta$-lactamase, iAmpC $\beta$-lactamase and ESBL among gram negative bacteria

\begin{tabular}{lcrc}
\hline \multirow{2}{*}{ Result } & \multicolumn{3}{c}{ No (\%) } \\
& AmpC & iAmpC & ESBL \\
\hline Positive & $75(52.1)$ & $12(8.3)$ & $60(41.7)$ \\
Negative & $69(47.9)$ & $132(91.7)$ & $84(58.3)$ \\
Total & $144(100)$ & $144(100)$ & $144(100)$ \\
\hline
\end{tabular}

The AmpC $\beta$-lactamase producing isolates showed increased resistance to antibiotics compared to nonAmpC $\beta$-lactamase producing isolates (Table-5). Among 144 GNB, 27 (18.7\%) organisms were resistance to more than 3 antibiotic classes (ciprofloxacin, cotrimoxazole $\&$ gentamicin) and they
(16.7\%) isolates showed combined AmpC $\beta$-lactamase and ESBL production (Table-4). The organisms isolated from in-patient department showed higher incidence of AmpC $\beta$-lactamase $(70.7 \%)$ when compared to organisms from out-patients $(36.7 \%)$. The difference was statistically significant with ( $p$ value 0.000 ).

Table - 4: Occurence of AmpC $\beta$-lactamase \& ESBL among gram negative bacteria

\begin{tabular}{lrrrr}
\hline \multirow{4}{*}{$\beta$-lactamase } & \multicolumn{2}{c}{ No $(\%)$} & \multicolumn{1}{c}{ Total } \\
& & Positive & \multicolumn{1}{c}{ Negative } \\
\hline \multirow{2}{*}{ AmpC } & Positive & $24(32 \%)$ & $51(68 \%)$ & $75(100 \%)$ \\
& Negative & $36(52.2 \%)$ & $33(47.8 \%)$ & $69(100 \%)$ \\
Total & & $60(58.3 \%)$ & $84(41.7 \%)$ & $144(100 \%)$ \\
\hline
\end{tabular}

were considered as multi drug resistant (MDR). Among the 27 MDR bacteria, 23 bacteria showed AmpC $\beta$ lactamase production $(\mathrm{p}<0.000)$. Cefoxitin resistance was seen in 82 isolates, among them 75 harboured AmpC $\beta$-lactamase enzymes and 7 showed resistance even without AmpC $\beta$-lactamase.

Table - 5: Antibiotic resistance among AmpC $\beta$-lactamase positive and AmpC $\beta$-lactamase negative GNB

\begin{tabular}{lrrrrrrrrrrrr}
\hline AmpC & AMC & CIP & COT & PIT & CPZ & CFS & GEN & AK & NIT & NA & NX & IPM \\
\hline Negative & 44.9 & 62.3 & 55.1 & 18.8 & 63.8 & 26.1 & 15.9 & 4.3 & 33.3 & 81.2 & 58.0 & 0.0 \\
Positive & 84.0 & 80.0 & 73.3 & 54.7 & 81.3 & 61.3 & 36.0 & 13.3 & 44.0 & 89.3 & 78.7 & 6.7 \\
\hline
\end{tabular}

\section{Discussion:}

The AmpC $\beta$-lactamase producing clinical isolates were resistant to available broad spectrum cephalosporins. Among the 900 samples submitted, 144 urine specimens yielded microorganisms. The occurrence of UTI in this study was $16 \%$. A study done by Taneja et al showed an isolation rate of $21.8 \%$ in their study ${ }^{\left[{ }^{[9]}\right.}$ The most common isolate (104/144) was Escherichia coli, followed by NFGNB and other members of Enterobacteriaceae. Multidrug resistance was found in $18.7 \%$ of isolates and similar finding was made by Taneja et al ${ }^{[9]}$ The increased MDR among the isolates may be due to the simultaneous carriage of drug resistance genes in resistance plasmids.

The AmpC $\beta$-lactamase production was found in $52.08 \%$ isolates. Cefoxitin resistance was found in 82 isolates and 75 were found to be positive by AmpC disk test with Tris-EDTA. The cefoxitin resistance among seven isolates without AmpC $\beta$-lactamase were assumed to be due to other resistance mechanism. At presently no approved guidelines for AmpC $\beta$ lactamase detection were found ${ }^{[1,2,4]} \mathrm{AmpC} \beta$-lactamase producing organisms will be positive in ESBL screening test but cannot be differentiated by confirmatory test. The AmpC $\beta$-lactamase production should be suspected when an isolate shows resistance to cefoxitin and third generation cephalosporins. But resistance to cefoxitin is not specific to AmpC $\beta$ lactamases as it can be found in carbapenem resistant isolates or isolates with loss of porin channel. ${ }^{[5]}$ Various methods are designed to detect AmpC $\beta$-lactamase. Modified three-dimensional test, AmpC disk test with Tris-EDTA and inhibitor based methods using cloxacillin or derivatives of boronic acid are widely used to detect AmpC $\beta$-lactamases. ${ }^{[3]}$ By using various 
combinations of cefotaxime and ceftazidime with clavualnic acid and boronic acid reliable differentiation of ESBL, AmpC $\beta$-lactamase or coproducers can be done in vitro. Phenotypic tests have limitations in technical expertisation, availability of chemicals and instruments. Currently gold standard test for identification of various families of AmpC $\beta$-lactamases is by multiplex PCR with six primer pairs. Importance of routine AmpC $\beta$-lactamase detection in clinical laboratory is a debate. ${ }^{[2,5]}$

In the present study, high proportion of uropathogens harbouring AmpC $\beta$-lactamase, ESBL and multi-drug resistance limits the treatment options. Some of the cephalosporins and aztreonam may appear sensitive AmpC $\beta$-lactamase producing isolates in vitro but fail to respond if they are used for therapy in vivo. ${ }^{[5]}$ Furthermore AmpC $\beta$-lactamase producing isolates are capable of developing resistance to carbapenems and can spread to other organisms horizontally.

Most of the isolates showed sensitive zones to imipenem and amikacin drugs. AmpC $\beta$-lactamase producing isolates often show resistance to multiple antibiotics and limits the treatment options. Cephalosporins, penicillins, betalactam-betalactamase inhibitor combinations should be avoided as they help in over expression of chromosomal AmpC $\beta$-lactamase resulting in poor clinical outcomes. Simultaneous occurence of ESBL and AmpC $\beta$-lactamase in an isolate further limit treatment options. ${ }^{[3,5]}$

The present study demonstrated higher proportion of uropathogens harbouring AmpC $\beta$-lactamase. An isolate possessing $\mathrm{ESBL}$, AmpC $\beta$-lactamase and multidrug resistance limit the treatment options. Routine identification of ESBL and AmpC $\beta$-lactamase is required to choose appropriate therapy. Imipenem can be used as empirical drug for treatment of complicated
UTI who are admitted to hospital.

\section{Financial Support and sponsorship: Nil Conflicts of interest: Nil}

\section{References:}

1. Gupta K, Sahm DF, Mayfield D, Stamm WE. Antimicrobial resistance among uropathogens that cause community acquired urinary tract infections in women: A nationwide analysis. Clin Infect Dis 2001; 33 : 89-94.

2. Philippon A, Arlet G, Jacoby GA. Plasmid-Determined AmpC-Type $\beta$-Lactamases. Antimicrob Agents Chemother 2002;46(1):1-11.

3. MJC Noyal, Menezes GA, Harish BN, Sujatha S, Parija SC. Simple screening tests for detection of carbapenemases in clinical isolates of nonfermentative Gram-negative bacteria. Indian J Med Res 2009; 129(6): 707-12.

4. Bartolini A, Frasson I, Cavallaro A, Richter SN, Palu G. Comparison of phenotypic methods for the detection of carbapenem non-susceptible Enterobacteriaceae. Gut Pathogens 2014;6:13 Pages 1 to 7.

5. Jacoby GA. AmpC $\beta$-Lactamases. Clin Microbiol Rev 2009; 22(1):161-82.

6. Barrow GI, Feltham RKA, editors. Cowan and Steels Manual for the Identification of Medical Bacteria. 3rd Ed. London: Cambridge University Press; 1993.

7. Clinical and Laboratory Standards Institute (CLSI). M100-S25. Performance standards for antimicrobial susceptibility testing; 25th informational supplement: Wayne, PA:CLSI;2015.

8. Black JA, Moland ES, Thomson KS. AmpC disk test for detection of plasmid-mediated AmpC $\beta$-lactamases in Enterobacteriaceae lacking chromosomal AmpC $\beta$ lactamases. J Clin Microbiol. 2005;43:3110-3.

9. Taneja N, Rao P, Arora J, Dogra A. Occurrence of ESBL \& Amp-C b-lactamases \& susceptibility to newer antimicrobial agents in complicated UTI. Ind J Med Res 2008; $127: 85-8$. 